\title{
CONTRIBUIÇÃO DAS CHUVAS DO PERÍODO DA TARDE EM BELÉM E POSSÍVEIS RELAÇÕES COM A NORMAL CLIMATOLÓGICA
}

\author{
MORAES, Dayse - dsmoraes1438@gmail.com \\ Universidade Federal de Campina Grande / UFCG \\ FILHO, Manoel - mano2442@yahoo.com.br \\ Universidade Federal de Campina Grande / UFCG
}

\begin{abstract}
RESUMO: O objetivo do estudo é avaliar qualitativamente e quantitativamente qual a contribuição das chuvas, do período da tarde (entre 1200 e 1800 horas), na cidade de Belém do Pará, em relação ao total mensal, determinadas através de análises estatísticas, a partir de dados horários pluviométricos, dos anos de 2003 a 2015 . Os resultados obtidos indicaram que as precipitações do período da tarde na cidade contribuem em média com $17 \%$ do total anual. O ciclo horário de marcha anual mostrou que as contribuições são diferentes para cada mês do ano. Nos meses menos chuvosos, essa porcentagem é de cerca de $30 \%$. As taxas de precipitação apresentaram picos máximos no horário das 1600 horas local (HL), influenciadas diretamente pela atuação da Zona de Convergência Intertropical (ZCIT), principal mecanismo gerador de chuvas em Belém, pelas Linhas de Instabilidade (LI) e pelos sistemas locais. Sugere-se que as oscilações interanuais da precipitação sazonal sejam principalmente decorrentes das variabilidades climáticas associadas aos eventos do Índice de Oscilação Sul e do Dipolo do Atlântico. Este estudo concluiu que a "famosa chuva da tarde" em Belém, tem grande contribuição para os totais mensais. Quanto à contribuição nos meses mais chuvosos (dezembro a maio), o mês de dezembro, apresentou contribuição de $26 \%$ em relação ao total mensal no horário das $1600 \mathrm{HL}$ e a menor, foi no mês de maio, com 14\%.
\end{abstract}

PALAVRAS-CHAVES: Belém, Precipitação, Contribuição, Ciclo horário, ZCIT.

\section{CONTRIBUCIÓN DE LAS LLUVAS DEL PERÍODO DE LA TARDE EN BELÉM Y POSIBLES RELACIONES CON LA NORMAL CLIMATOLÓGICA}

RESUMEN: El objetivo del estudio es evaluar cualitativamente y cuantitativamente cuál es la contribución de las lluvias, del período de la tarde (entre 1200 y 1800 horas), en la ciudad de Belém do Pará, en relación al total mensual, determinadas a través de análisis estadísticos, a partir de datos horarios pluviométricos, de los años 2003 a 2015. Los resultados obtenidos indicaron que las precipitaciones de la tarde en la ciudad contribuyen en promedio con el $17 \%$ del total anual. El ciclo horario de marcha anual mostró que las contribuciones son diferentes para cada mes del año. En los meses menos lluviosos, este porcentaje es de alrededor del 30\%. Las tasas de precipitación presentaron picos máximos en el horario de las 1600 horas local (HL), influenciadas directamente por la actuación de la Zona de Convergencia Intertropical (ZCIT), principal mecanismo generador de lluvias en Belém, por las Líneas de Inestabilidad (LI) y por los sistemas locales. Se sugiere que las oscilaciones interanuales de la precipitación estacional se deban principalmente a las variabilidades climáticas asociadas a los eventos del Índice de Oscilación Sur y del Dipolo del Atlántico. Este estudio concluyó que la "famosa lluvia de la tarde" en Belém, tiene gran contribución a los totales mensuales. En cuanto a la contribución en los meses más lluviosos (diciembre a mayo), el mes de diciembre, presentó una contribución del $26 \%$ en relación al total mensual en el horario de las $1600 \mathrm{HL}$ y la menor, fue en el mes de mayo, con el $14 \%$.

PALABRAS CLAVE: Belém, Precipitación, Contribución, Ciclo horario, ZCIT

\section{CONTRIBUTION OF THE AFTERNOON RAINING IN BELEM AND POSSIBLE RELATIONS WITH NORMAL CLIMATOLOGY}

ABSTRACT: The objective of the study is to evaluate qualitatively and quantitatively the contribution of rainfall, in the afternoon (between 1200 and 1800 hours), in the city of Belém do Pará, in relation to the monthly total, determined through statistical analyzes, 
based on data The results indicate that rainfall in the afternoon in the city contributes on average $17 \%$ of the annual total. The annual cycle of the march showed that the contributions are different for each month of the year. In the less rainy months, this percentage is around $30 \%$. The precipitation rates presented maximum peaks in the 1600 (HL) local time, influenced directly by the Intertropical Convergence Zone (ZCIT), the main rainfall generating mechanism in Belém, by the Instability Lines (LI) and by the local systems. It is suggested that the interannual oscillations of the seasonal precipitation are mainly due to the climatic variabilities associated to the events of the South Oscillation Index and the Atlantic Dipole. This study concluded that the "famous afternoon rain" in Belém has a large contribution to the monthly totals. As for the contribution in the rainy months (December to May), the contribution of $26 \%$ in relation to the monthly total in the hours of $1600 \mathrm{HL}$ and the lowest, was $14 \%$ in May.

KEYWORDS: Belem, Precipitation, Contribution, Time series, ITCZ.

\section{CONTRIBUTION DE LA PLUIE DE L'APRĖS-MIDI EN BELÉM ET RELATIONS POSSIBLES AVEC LA CLIMATOLOGIE NORMALE}

RESUME: L'objectif de l'étude est d'évaluer qualitativement et quantitativement la contribution de la pluie, l'après-midi (entre 1200 et 1800 heures) dans la ville de Belém do Pará, par rapport au total mensuel déterminé par l'analyse statistique, à partir des données Les résultats indiquent que les précipitations dans l'après-midi dans la ville contribuent en moyenne à $17 \%$ du total annuel. Le cycle annuel de la marche a montré que les contributions sont différentes pour chaque mois de l'année. Dans les mois moins pluvieux, ce pourcentage est d'environ $30 \%$. Les taux de précipitation ont montré pic maximum dans le temps de 1600 heure locale $\mathrm{HL}$, directement influencée par la performance de la zone de convergence intertropicale (ZCIT), les principales pluies mécanisme de production à Bethléem, les lignes d'instabilité (LI) et les systèmes locaux. Il est suggéré que les fluctuations interannuelles des précipitations saisonnières sont principalement dues à la variabilité climatique associée aux événements de l'indice d'oscillation australe et l'Dipole Atlantique. Cette étude a conclu que la «fameuse pluie de l'après-midi» à Belém contribue largement aux totaux mensuels. Quant à la contribution dans les mois les plus pluvieux (décembre à mai), le mois de Décembre, a montré la contribution de $26 \%$ et le total mensuel au moment du $1600 \mathrm{HL}$ et le plus bas était en mai, avec $14 \%$.

MOT CLÉS: Bethléem, Précipitation, Contribution, Série chronologique, ZCIT.

\section{INTRODUÇÃO}

A cidade de Belém, capital do estado do Pará é caracterizada por apresentar altos índices pluviométricos devido à sua proximidade com a linha do equador. O clima da região é influenciado por sistemas atmosféricos de meso escala (como Brisa Marítima e Linhas de Instabilidade) (COHEN, 1989; GARSTANG et al., 1994; COHEN et al., 1995; ALCÂNTARA et al., 2011; ALCÂNTARA et al., 2014; MATOS e COHEN, 2016; OLIVEIRA et al.,2016), de escala sinótica (Distúrbio Ondulatório de Leste e Vórtices Ciclônicos de Altos Níveis) (LIEBMANN, 1990; ASNANI, 1993; MOTA, 1997; SOUZA et al., 2000; CAVALCANTE et al., 2003) e de grande escala (Zona de Convergência Intertropical) (KOUSKY e KAGANO, 1981; NIMER, 1989; SANTOS, 2006; CAVALCANTE et al., 2009; FERREIRA et al., 2015) que podem provocar grandes volumes de chuva (FIGUEREDO e MOTA, 2010).

Em Belém, é tradicional a pergunta: "Você vai sair antes ou depois da chuva?". Quem não a conhece pode até se assustar, mas ela é bastante comum na região. Na cidade chove praticamente todos os dias e quase com horário marcado (às 15 horas). Porém, essa chuva vem sendo alterada, principalmente durante o verão amazônico (setembro a novembro) quando normalmente chove 
pouco e acoplado a isso outros fenômenos climáticos, como o El Niño e outro mecanismo que influência o regime de chuva na região é o Gradiente Meridional da Temperatura da Superfície do Mar (TSM) do Atlântico Tropical (ANDREOLI e KAYANO, 2007; FERREIRA et al., 2015).

Mesmo sendo comum imaginar que as chuvas permaneçam no mesmo horário, estatisticamente, tal pontualidade não é observada, em aproximadamente $30 \%$ dos dias do ano não chove em Belém, e $40 \%$ dos dias considerados chove de 0 a 0,9 mm/dia. A chuva não se apresenta, mas em hora marcada. Ocorreu variabilidade nos horários, uma variabilidade sazonal. Nechet (1984b, 1997), observou que no período mais chuvoso, a partir da segunda quinzena de dezembro a meados de maio, as precipitações ocorrem normalmente entre 14 e 15 horas, a partir desse horário, observa-se um deslocamento para mais tarde, ou seja, no final do período chuvoso (junho, julho, agosto) entre 17 e 21 horas e entre setembro e dezembro, gradativamente, começa a voltar para o meio da tarde, entre 15 e 16 horas.

Um dos sistemas que influenciam o ciclo diurno das chuvas são as linhas de instabilidade (LI) que se formam ao longo da costa amazônica, com sua gênese associada à circulação de brisa marítima (BM) (KOUSKY, 1980), sendo responsáveis por $45 \%$ da precipitação no leste do Pará e na região central da Amazônia, durante a estação chuvosa (COHEN et al., 1995; JANOWIAK et al., 2005).

Recentemente, Matos e Cohen (2016), denominaram um sistema convectivo, observado pela primeira vez em Belém, durante a campanha do projeto CHUVA - Cloud processes of the main precipitation systems in brazil: A contribUtion to cloud resolVing modeling and to the gpm (globAl precipitation measurement) (MACHADO et al., 2014), como micro linhas de instabilidade fluvial (MLF), devido sua formação está associada à circulação de brisa fluvial (BF). O sistema, proveniente da baía do Marajó, provocou precipitação desde sua gênese sobre a região de Belém até a margem oeste da baía, aumentando a intensidade da precipitação nessa região.

Outro estudo sugere a existência de brisa fluvial, na região de Belém, ao longo do ano, com horário de ocorrência de 1200 a 0000 UTC, caso observacional de Germano et al. (2017). Os autores também confirmaram a influência da circulação de BM, gerando uma precipitação cumulativa durante os horários de 1500-1800 UTC na costa e no interior de 2100-0000 UTC.

Embora as análises de chuva para a região de Belém, que se caracteriza por ser uma área de elevada pluviosidade e sem período seco, tenham sido realizadas nos últimos anos (como por exemplo, LOUREIRO et al., 2014), há carência de estudos para essa região. Portanto, o objetivo do presente trabalho é fornecer informações inéditas sobre o comportamento da precipitação em escala temporal horária e quantificar qual a importância da "famosa chuva da tarde" de Belém do Pará no total médio mensal pluviométrico.

\section{MATERIAL E MÉTODOS}

\section{ÁREA DE ESTUDO}

A área de estudo abrange o estado do Pará (norte do Brasil) (Figura 1a) e especificamente a sua capital Belém. O Pará é o segundo maior estado do 
Brasil em extensão territorial com 1.248.042,515 km², dividido em 144 municípios. Os principais rios são o rio Amazonas, rio Tapajós, rio Tocantins, rio Xingu e o rio Pará (Figura 1b). Segundo o INMET (Instituto Nacional de Meteorologia), a cidade de Belém, banhada pelo rio Guamá e pela baía do Guajará (capturada por satélite - Figura 1c), é considerada a capital mais chuvosa do Brasil, com índice pluviométrico anual de 2921,9 mm/ano, possui uma área de $1.064,918 \mathrm{~km}^{2}$, uma altitude de dez metros acima do nível médio do mar, trinta e nove ilhas em seu território, situadas no oceano Atlântico e limita-se com os municípios de Ananindeua, Marituba, Santa Barbara do Pará e Barcarena, além da baía do Marajó.
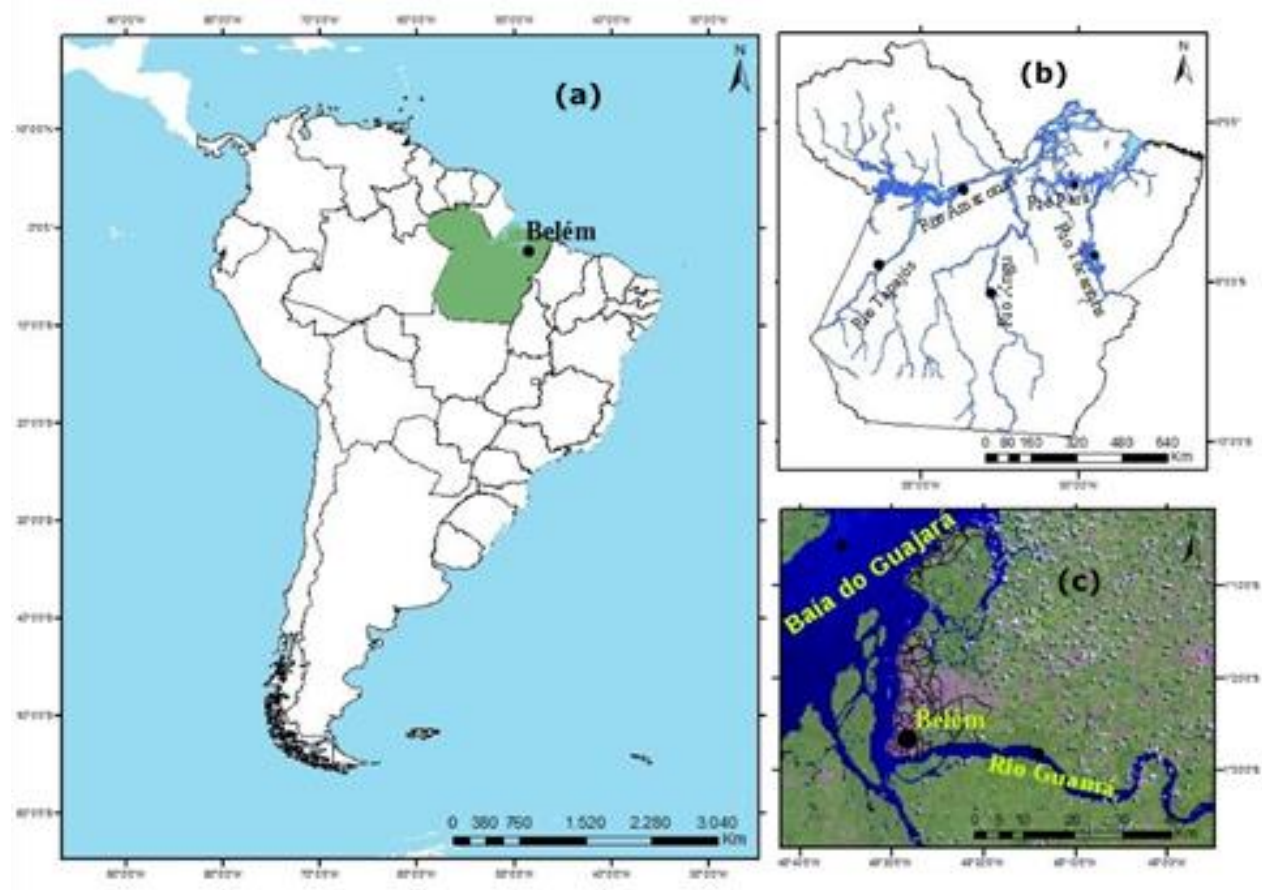

Figura 1 - Mapa do Brasil, em destaque, na cor verde, o estado do Pará. (b) Mapa do estado do Pará e seus principais rios e afluentes. (c) Imagem de satélite da cidade de Belém, as margens do rio Guamá e da baía do Guajará.

\section{CLIMATOLOGIA DA REGIÃO}

O clima em Belém tem influência direta da floresta amazônica, de seus rios e de sua proximidade com o oceano Atlântico. Sendo uma região onde as chuvas são constantes, a cidade apresenta duas estações: estação chuvosa (dezembro a maio) e estação menos chuvosa (junho a novembro) (Figura 2), conforme descrito por Nechet (1984a), Figueroa e Nobre (1990), Bastos et al., (2002) e Oliveira et al., (2003).

Em geral, a época chuvosa inicia-se em dezembro e tem duração de cinco a seis meses. Os maiores índices pluviométricos são observados entre os meses de janeiro a abril, com o mês de março apresentando os maiores valores (438 mm/mês), fato que é justificado pela intensificação dos efeitos locais associados à presença da ZCIT, causando os trimestres mais chuvosos de 
março, abril e maio e de dezembro, janeiro e fevereiro. A época menos chuvosa, compreendem os meses de junho até novembro, onde predominam as chuvas de caráter convectivo na forma de pancadas de chuvas isoladas, sendo que o período de menor precipitação mensal climatológica ocorre entre os meses de agosto (127 mm/mês) e novembro (111 mm/mês) (ALBUQUERQUE et al., 2010) (Figura 2).

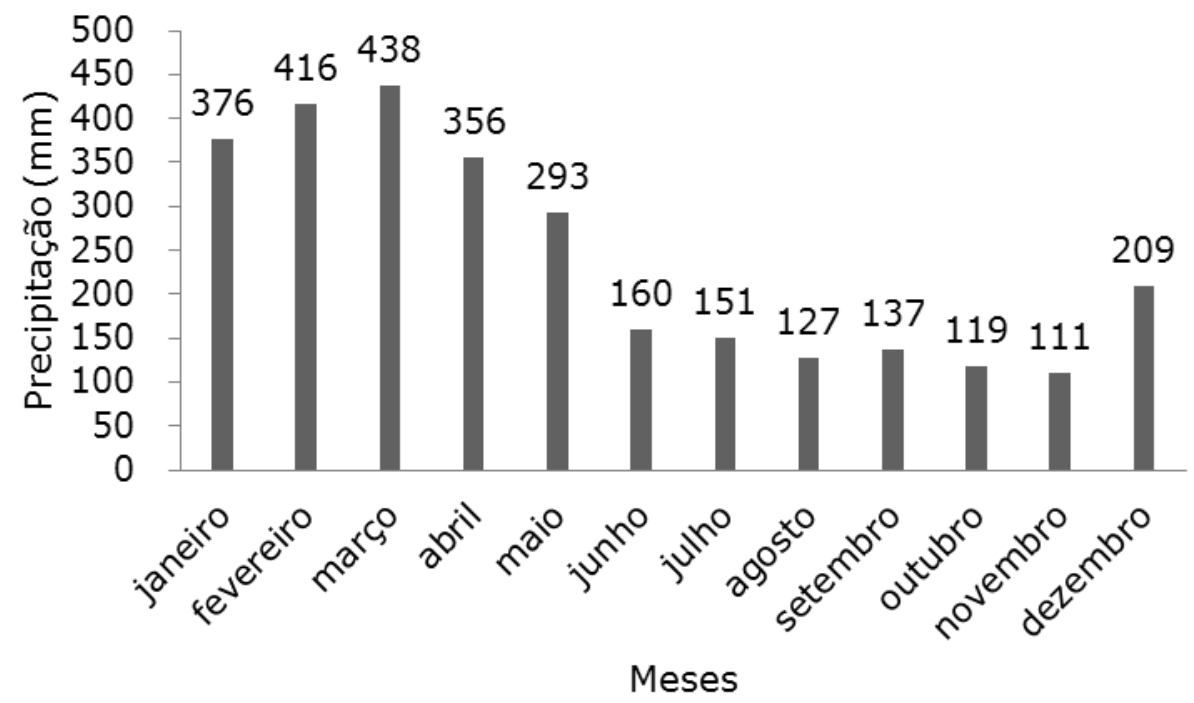

Figura 2 - Médias climatológicas da precipitação mensal (média 1961-1990) representativas de Belém do Pará para os meses de janeiro a dezembro. Adaptado pelo autor. Fonte de dados: INMET.

\section{DESCRIÇÃO DOS DADOS}

Foram utilizadas séries temporais de precipitações horárias para a estação meteorológica automática (EMA), localizada na sede do $2^{\circ}$ Distrito de

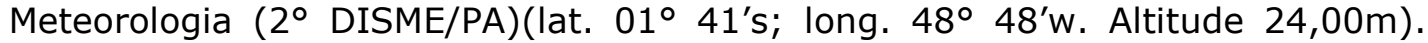
Período utilizado foi de 2003 a 2015. Esses anos foram escolhidos devido à mesma ter sido instalada no ano de 2003. Os acumulados observados, por exemplo, são acumulados das 1200 UTC (0900 HL) às 1259 UTC (0959 HL), o qual corresponderia ao horário das 1300 UTC (1000 HL) e assim sucessivamente. A EMA utilizada é composta de uma unidade de memória central ("data logger"), de fabricação finlandesa, marca Vaisala, modelo MAWS 301, usadas como padrão para a rede de EMA do INMET. O pluviômetro é do tipo Tipping Bucket (Vaisala).

Para comparação das fases do ENOS com a variabilidade interanual da precipitação foi utilizado o índice do Niño Oceânico (INO) do "Climate Prediction Center" da "National Oceanic and Atmospheric Administratation" (NOAA), disponível

www.cpc.noaa.gov/products/analysis_monitoring/ensostuff/ensoyears.shtml. Este índice define as fases com base na ATSM em intensidade fraca (ATSM de $0,5^{\circ} \mathrm{C}$ a $\left.0,9^{\circ} \mathrm{C}\right)$, moderada $\left(1,0^{\circ} \mathrm{C}\right.$ a $\left.1,4^{\circ} \mathrm{C}\right)$ e forte $\left(\geq 1,5^{\circ} \mathrm{C}\right)$.

\section{ESTATÍSTICA DESCRITIVA}


Por meio da estatística descritiva é possível obter características essenciais para a formação de histograma de frequência relativas de uma amostra de dados hidrológicos (NAGHETTINI e PINTO, 2007). Para este estudo foram calculadas as medidas de tendência central.

\section{MEDIDA DE TENDÊNCIA CENTRAL}

A média é a medida da posição mais frequentemente usada e tem um significado teórico importante na estimativa de amostras. É calculada pela seguinte fórmula:

$$
\dot{X}=\frac{x_{1} \ldots \ldots \ldots \ldots \ldots \ldots \ldots \ldots x_{n}}{N}=\frac{1}{N} \sum_{1}^{n} x_{i}
$$

Em que $X$ é a média; $N$ é o tamanho da amostra e $x_{i}$ até $x_{n}$ são os somatórios da amostra.

\section{RESULTADOS E DISCUSSÃO}

\subsection{MEDIDAS DE TENDÊNCIA CENTRAL E CONTRIBUIÇÃO PLUVIAL}

Foi efetuada uma abordagem climática das chuvas, para o cálculo da média aritmética, proporcionando uma visão quantitativa das contribuições percentuais de cada hora do dia das precipitações nos anos de 2003 a 2015. A Figura 3 mostra o comportamento do ciclo horário da precipitação na cidade de Belém-PA e a média percentual da contribuição pluviométrica do total mensal dos anos de 2003 a 2015.

Visto que Belém possui característica bem definida, como o aquecimento superficial no decorrer do dia e maior quantidade de precipitação no fim da tarde e início da noite, a figura mostrou maiores contribuições entre 1500 e $1800 \mathrm{HL}$ (média de 14\%). Destaque na figura é que entre o final da noite, durante a madrugada e no inicio da manhã (de 2200 as $1200 \mathrm{HL}$ ) a média de contribuição é mínima, alcançando valores abaixo de $2 \%$. No começo da tarde (1300 HL) essa contribuição aumenta gradativamente e as $1600 \mathrm{HL}$ alcança o pico máximo $(17 \%)$, nesse horário há condições propicias para o desenvolvimento de nuvens cumuliformes (cúmulos congestus e Cbs, isto é, com dimensão vertical acentuada), formando, então, fortes precipitações principalmente no período da tarde, aumentando, assim, a contribuição, e a partir das $1700 \mathrm{HL}$, observa-se que esses valores tem um decréscimo. 


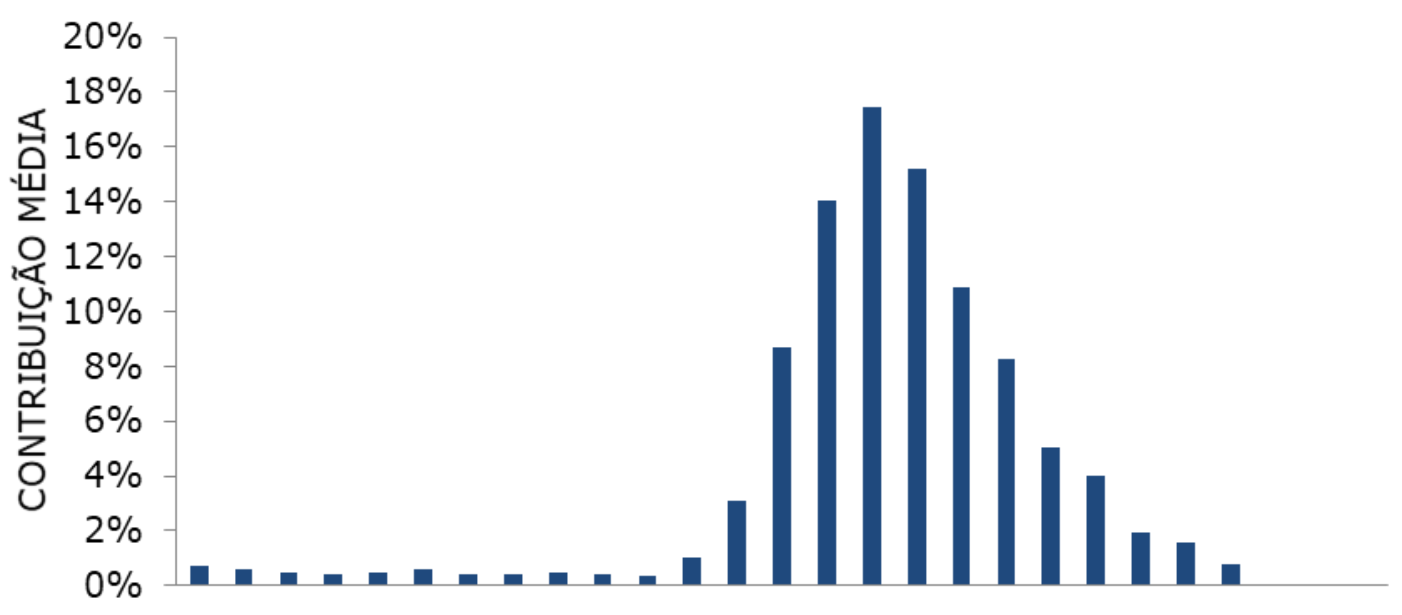

123456789101112131415161718192021222324

HORA LOCAL

Figura 3 - Média percentual de precipitação da contribuição diária referente aos anos de 2003 a 2015.

\subsection{ANÁlise dA CONTRIbUiçÃo percentuAl dos meses mais CHUVOSOS}

A Figura 4 mostra a variação horária da média percentual da contribuição pluviométrica do total mensal para Belém-PA, nos anos de 2003 a 2015, referentes aos meses mais chuvosos.

Nota-se na figura, que em todos os meses as maiores contribuições foram nos horários vespertinos com valores acima de $10 \%$ e um pico máximo no horário em torno das $1600 \mathrm{HL}$. Esse máximo pode ser justificado pela atuação da ZCIT e pelos efeitos de meso escala, como as LI que se propagam para oeste como uma linha de Cbs e associadas à BM, formada no período da tarde, provoca chuvas intensas, com mais frequência entre abril e agosto, e também, à formação de MLF, que ocorrem entre 1200 e $1430 \mathrm{HL}$, e entre 1430 e 2000 HL, a formação de micro linhas de instabilidade marítima.

As características observadas seguem do mesmo modo em todos os meses com valores abaixo de $2 \%$ desde a madrugada até o meio da tarde, com picos ao final da tarde e no início da noite. Deste modo, a expectativa de ocorrência de precipitação durante a manhã é baixíssima. Por outro lado, a partir das $1500 \mathrm{HL}$ o aquecimento superficial e a circulação de brisa no litoral deixa a atmosfera favorável à formação de nuvens com topos elevados, aumentando a probabilidade de ocorrência de precipitação intensa e rápida.

Devido às chuvas retornarem para o período da tarde, no mês de dezembro, o pico máximo apresenta-se com maior valor $(26 \%)$ e no mês de maio, seria menor, correspondendo a $14 \%$. Há um pequeno aumento nas contribuições nos meses de abril e maio, no período da noite em relação aos outros meses que na figura observa-se abaixo de $2 \%$. Isso pode está relacionado ao deslocamento da ZCIT para o HN nesses meses. 

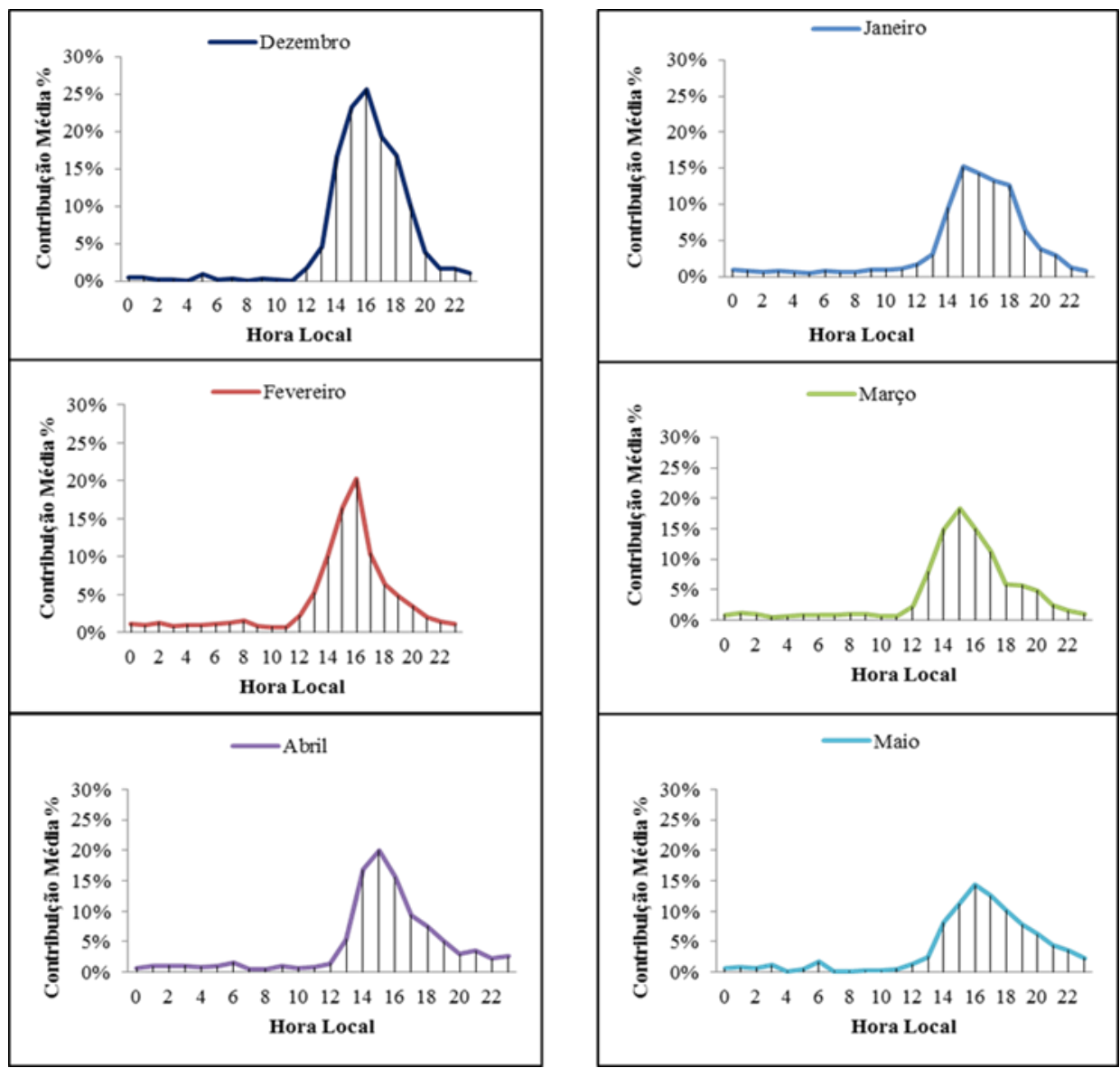

Figura 4 - Variação horária da média percentual da contribuição do total pluviométricos mensais, referentes ao período mais chuvoso (dezembro a maio) em Belém.

\subsection{ANÁlise DA CONTRIBUIÇÃo PERCENTUAL HORÁRIA DA PRECIPITAÇÃO NOS MESES MENOS CHUVOSOS}

Apresenta-se, na Figura 5, o ciclo horário da média percentual da contribuição do total pluviométrico, nos meses menos chuvosos em Belém, dos anos de 2003 a 2015. Observa-se que de setembro a novembro, considerado o período de estiagem, a precipitação geralmente é provocada pelos fenômenos de mesoescala (BASTOS et al., 2002), com isso as maiores contribuições estão no período da tarde, correspondendo a $26 \%$ do total pluviométrico e as contribuições à noite alcançam valores abaixo de $2 \%$, semelhante à figura 4 . 0 ciclo horário apresenta dois máximos, o primeiro no mês de setembro e o segundo em novembro com um percentual médio de $25 \%$ do total pluviométrico, no horário entre $1600 \mathrm{HL}$ e $1700 \mathrm{HL}$. Nos outros meses (junho, julho, agosto e outubro) essa contribuição ficou em torno de $15 \%$.

Nos meses de junho, julho e agosto notam-se uma contribuição um pouco maior no período da noite, em torno de $5 \%$, isso deve está relacionado aos SCM, que são sistemas que se originam no período da noite, além de MLF (MATOS e COHEN, 2016) que tem frequência no mês de agosto e o maior número de ocorrências de LI visto entre os meses de abril a agosto, neste período destaca-se a formação de LIP (COHEN et al.,1989). 
Além dessa curva apresentar contribuições no período noturno, nos meses de junho, julho e agosto, meses do fim do período chuvoso, as mesmas podem ter sido provocadas, também, por efeitos locais, como as BT e BM e por $\mathrm{DOL}$, visto que a maior frequência desses sistemas é principalmente no período da tarde e consequentemente as maiores contribuições ficou nesse período, e assim, a menor contribuição no período noturno, é observada nos meses de outubro, novembro e dezembro, quando a ZCIT encontra-se mais ao norte da sua climatologia.
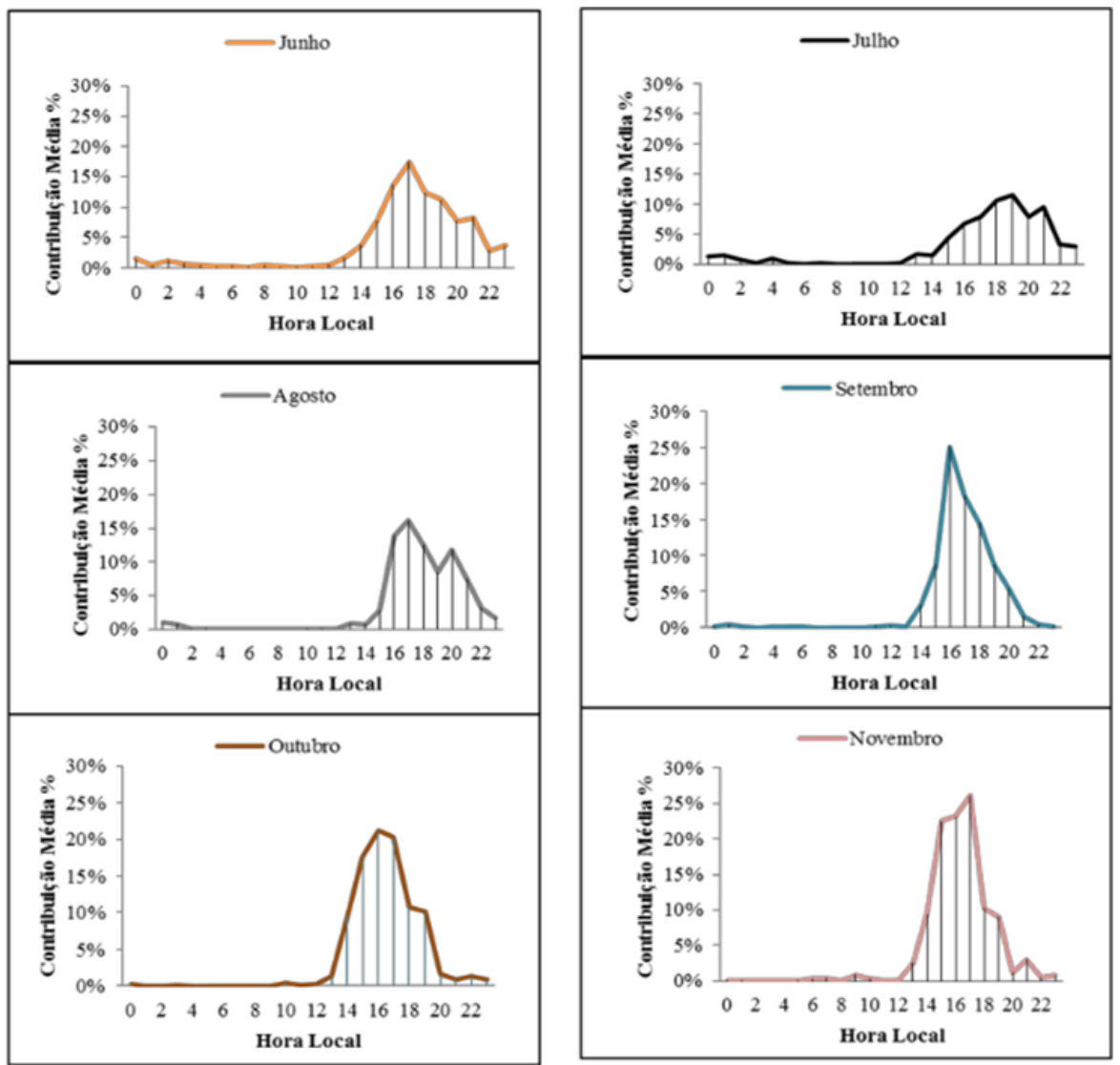

Figura 5 - Variação horária da média percentual da contribuição do total mensal referentes ao período menos chuvoso (junho a novembro) em Belém.

\subsection{ANÁLISE INTERANUAL E MENSAL DA PRECIPITAÇÃO PARA OS ANOS DE 2003 A 2015 E OS EVENTOS ENOS NA PRECIPITAÇÃO DE BELÉM - PA.}

A Figura 6 apresenta a variabilidade interanual do acumulado da precipitação durante os anos de 2003 a 2015. Notou-se que esse acumulado variou de $2500 \mathrm{~mm} /$ ano a $3800 \mathrm{~mm} /$ ano. Os anos que apresentaram maiores acumulados de precipitação foram em 2006 com 3663,8mm, 2013 apresentou 3775,6 mm, 2011 com 3592,2 mm e 2012 com 3563 mm de chuva. Excetuando os anos de 2006 e 2013 que foram anos neutros, os anos de 2011 e 2012 estiveram sob a influência do fenômeno LN que aumenta as chuvas na cidade de Belém. Os menores volumes de precipitação ocorreram nos anos de 2003 com 2769,4 mm de chuva e 2004 com 2940,2 mm. Esses anos estiveram sob a 
influência do fenômeno EN que inibe as chuvas em Belém-PA (MONTEIRO e MOTA, 2010). Esses resultados foram identificados pelo CPC/NOAA (2010), em que o ENOS foi negativo nos anos de 2003, 2004-2005 e 2006-2007(início). E nos anos de 2007(final)- 2008 o índice foi positivo configurando um evento LN, conforme a Tabela 1.

Tabela 1 - Ocorrência de eventos de El Niño e La Niña durante o período de 2003 a 2015.

\begin{tabular}{|c|c|c|c|c|c|c|c|c|c|c|c|c|}
\hline Anos & DJF & JFM & FMA & MAM & AMJ & MJJ & JJA & JAS & ASO & SON & OND & NDJ \\
\hline 2003 & 0.9 & 0.7 & 0.4 & 0.0 & -0.2 & -0.1 & 0.1 & 0.2 & 0.2 & 0.3 & 0.3 & 0.3 \\
\hline 2004 & 0.3 & 0.3 & 0.2 & 0.1 & 0.2 & 0.3 & 0.5 & 0.6 & 0.7 & 0.7 & 0.6 & 0.7 \\
\hline 2005 & 0.7 & 0.6 & 0.5 & 0.5 & 0.3 & 0.2 & 0.0 & -0.1 & 0.0 & -0.2 & -0.5 & -0.7 \\
\hline 2006 & -0.7 & -0.6 & -0.4 & -0.2 & 0.0 & 0.0 & 0.1 & 0.3 & 0.5 & 0.7 & 0.9 & 0.9 \\
\hline 2007 & 0.7 & 0.4 & 0.1 & -0.1 & -0.2 & -0.3 & -0.4 & -0.6 & -0.9 & -1.1 & -1.3 & -1.3 \\
\hline 2008 & -1.4 & -1.3 & -1.1 & -0.9 & -0.7 & -0.5 & -0.4 & -0.3 & -0.3 & -0.4 & -0.6 & -0.7 \\
\hline 2009 & -0.7 & -0.6 & -0.4 & -0.1 & 0.2 & 0.4 & 0.5 & 0.5 & 0.6 & 0.9 & 1.1 & 1.3 \\
\hline 2010 & 1.3 & 1.2 & 0.9 & 0.5 & 0.0 & -0.4 & -0.9 & -1.2 & -1.4 & -1.5 & -1.4 & -1.4 \\
\hline 2011 & -1.3 & -1.0 & -0.7 & -0.5 & -0.4 & -0.3 & -0.3 & -0.6 & -0.8 & -0.9 & -1.0 & -0.9 \\
\hline 2012 & -0.7 & -0.5 & -0.4 & -0.4 & -0.3 & -0.1 & 0.1 & 0.3 & 0.3 & 0.3 & 0.1 & -0.2 \\
\hline 2013 & -0.4 & -0.4 & -0.3 & -0.2 & -0.2 & -0.2 & -0.3 & -0.3 & -0.2 & -0.3 & -0.3 & -0.3 \\
\hline 2014 & -0.5 & -0.5 & -0.4 & -0.2 & -0.1 & 0.0 & -0.1 & 0.0 & 0.1 & 0.4 & 0.5 & 0.6 \\
\hline 2015 & 0.6 & 0.5 & 0.6 & 0.7 & 0.8 & 1.0 & 1.2 & 1.4 & 1.7 & 2.0 & 2.2 & 2.3 \\
\hline & & \multicolumn{3}{|c|}{ La Niña } & \multicolumn{3}{|c|}{ Normal } & \multicolumn{3}{|c|}{ El Niño } & & \\
\hline
\end{tabular}

Fonte: CPC/NCEP/NOAA (2016).

Os eventos ocorridos em 2006 e 2013 estiveram entre os anos de maiores volumes de precipitação anuais, porém o fenômeno ENOS estava em neutralidade, indicando que existiu influencia de outro padrão para a variação acentuada da precipitação nestes anos. Kayano e Capistrano (2013) e Ferreira et al. (2015) evidenciaram um padrão de anomalias da TSM sobre o Atlântico Tropical, comumente chamado de Dipolo do Atlântico, e que este padrão esta associado a mudanças nos valores de precipitação sobre o Norte e Nordeste do Brasil. Conforme a Tabela 2, o ano de 2006 esteve com o Dipolo negativo, ou seja, água mais aquecidas no Atlântico Sul, favorável à chuva na região Norte. E no ano de 2013, o Dipolo estava positivo (águas mais aquecidas no Atlântico Norte), o que não favorece as chuvas sobre a região, porém, como mostra a Figura 7 (inferior), há anomalias positivas de TSM próximo à costa da região Norte nestes anos, favorecendo o possível deslocamento da ZCIT, e com isso, a formação de outros sistemas, estando assim, associado ao aumento da precipitação.

Já nos anos de 2007-2008 foi identificado pelo CPTEC/INPE (2010) um evento LN de forte intensidade, conforme mostrando pelo IOS negativo na 
Tabela 1, entretanto no ano de 2007, o cenário de precipitação não foi muito diferente do ano de 2011 e 2012, pois a precipitação em Belém, mostradas na Figura 6, foi menor, como deveria acontecer em anos de LN, assim pode-se afirmar que esse fenômeno possuiu pouca influência no regime pluviométrico na cidade de Belém. Essa região também experimenta a ocorrência de sistemas atmosféricos de meso escala, como SCMC que são facilmente observados por satélite. Em 2011, ano de LN, os SCMC maiores foram mais frequentes e restritos à foz do rio Tocantins e da baía de Marajó, conforme analisado por Sodré (2013). Embora o ENOS tenha um papel importante na interação entre a TSM e a precipitação da Amazônia, ele não é o único modulador da precipitação nesta região, por isso que nem todos os eventos de maiores/menores precipitações estão associados a este padrão.

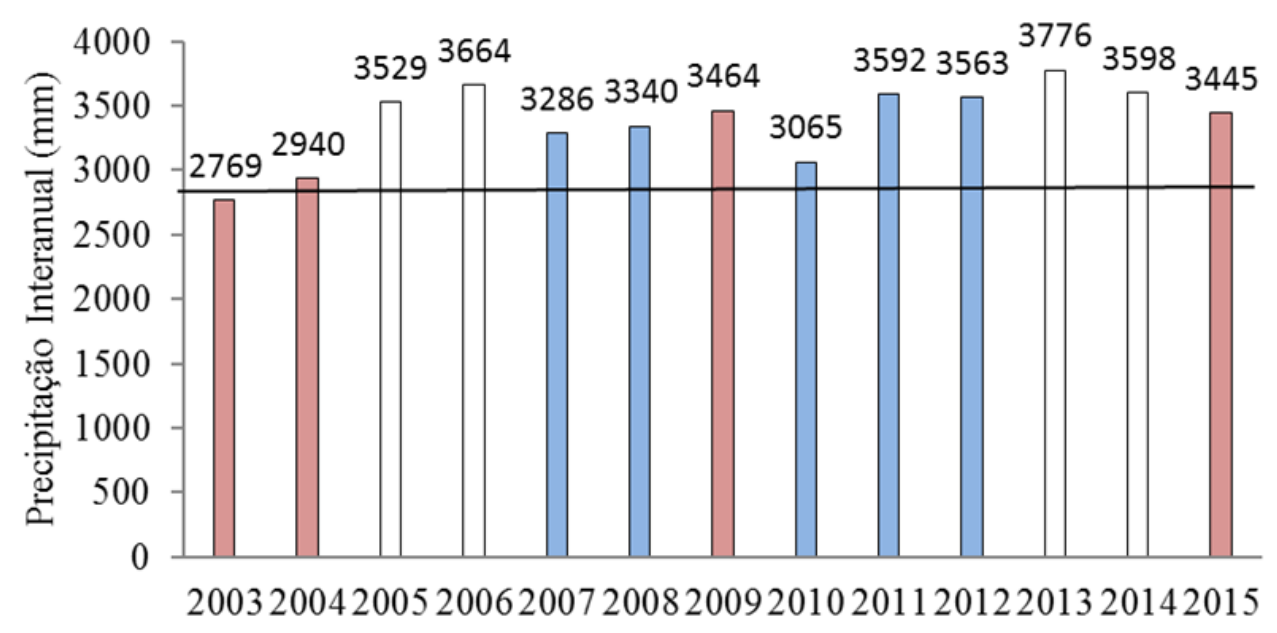

Anos

Figura 6 - Variabilidade interanual da precipitação em Belém para 2003-2015. As barras na cor vermelha representam os anos que estiveram sob a influência do fenômeno El Niño, as barras em azul, anos sob a influência da La Niña e as brancas foram os anos que não estiveram sob a influência do ENOS. Linha continua é a média pluviométrica anual.

Tabela 2 - Anos de ocorrência de eventos de Dipolo Positivo e Dipolo Negativo durante o período de 2003 a 2015.

\begin{tabular}{|c|c|}
\hline \multicolumn{2}{|c|}{ Oceano Atlântico } \\
DIPOLO (+) & DIPOLO (-) \\
ANOS & ANOS \\
$2004,2005,2007,2010,2012$, & $2003,2006,2008,2009,2011,2014$, \\
2013 & 2015 \\
\hline
\end{tabular}



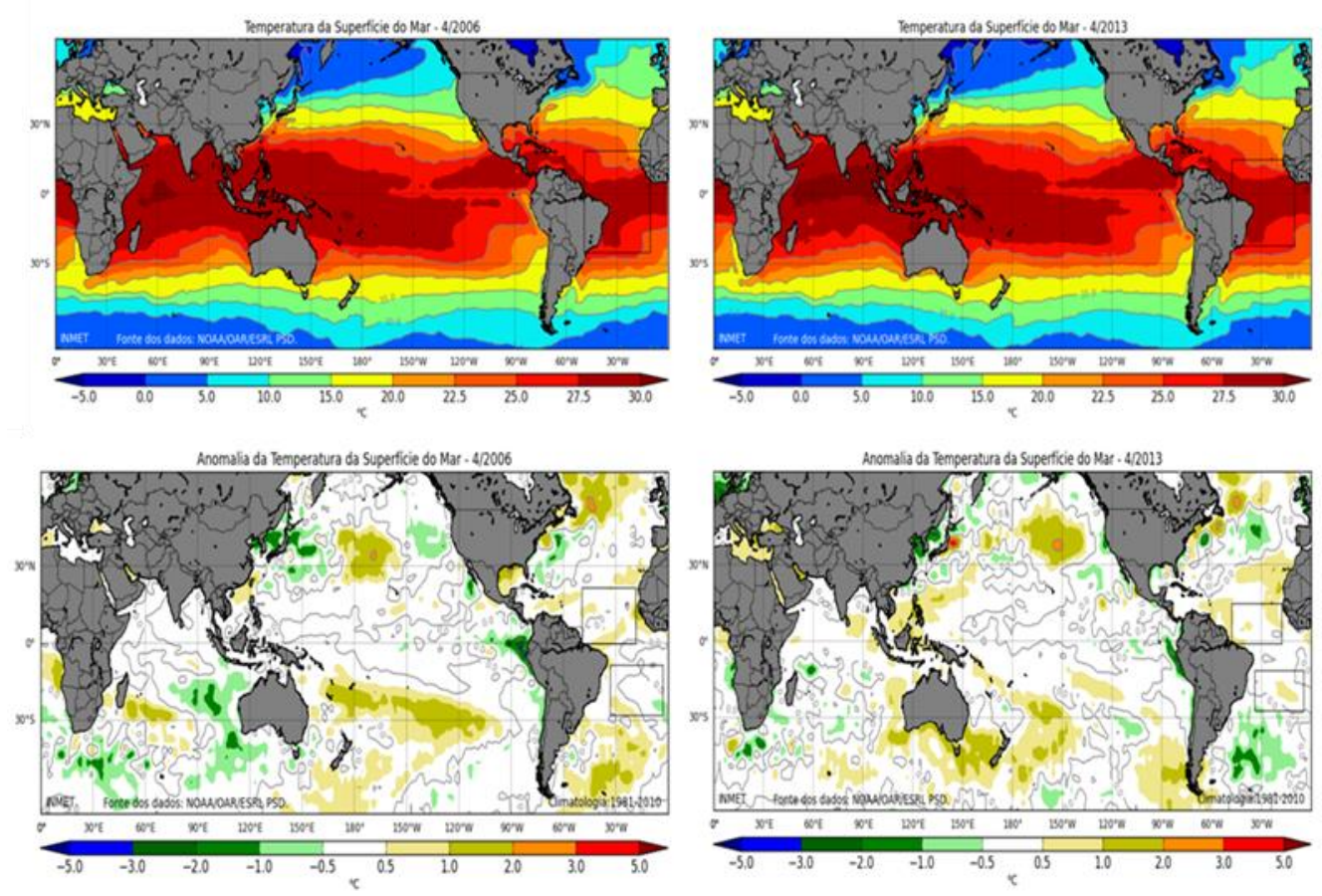

Figura 7 - Temperatura da superfície do mar (superior) e Anomalias da temperatura da superfície do mar (inferior), no mês de abril de 2006 e 2013. Fonte: INMET.

Analisando a precipitação média mensal em $\mathrm{mm}$. dia -1 nos anos de 2003-2015 (Figura 8) é possível observar que nos meses de dezembro a maio, meses mais chuvosos, ocorreu uma média mensal de 13,2 mm. dia -1 de precipitação $(72,7 \%$ do total da média mensal). Os meses com maior precipitação foram março e abril, a média mensal desses dois meses foi de 16,2 $\mathrm{mm}$. dia -1 de chuva $(29,7 \%$ do total da média mensal). Enquanto que nos meses menos chuvosos (junho a novembro) a média mensal foi de $5 \mathrm{~mm}$. dia-1, o que representou $27,3 \%$ do total da média mensal.

A variabilidade da precipitação entre os dois períodos (chuvoso e menos chuvoso) mostra a influência dos fenômenos de meso e grande escala no aumento e na redução de precipitação. O volume de precipitação, no período chuvoso, está relacionado ao período de atuação da ZCIT e da Oscilação de Madenn-Julian (OMJ) na região (DE SOUZA e AMBRIZZI, 2006). Enquanto que o período menos chuvoso, as LI são responsáveis por cerca de $45 \%$ da ocorrência das chuvas (COHEN et al., 1995). 


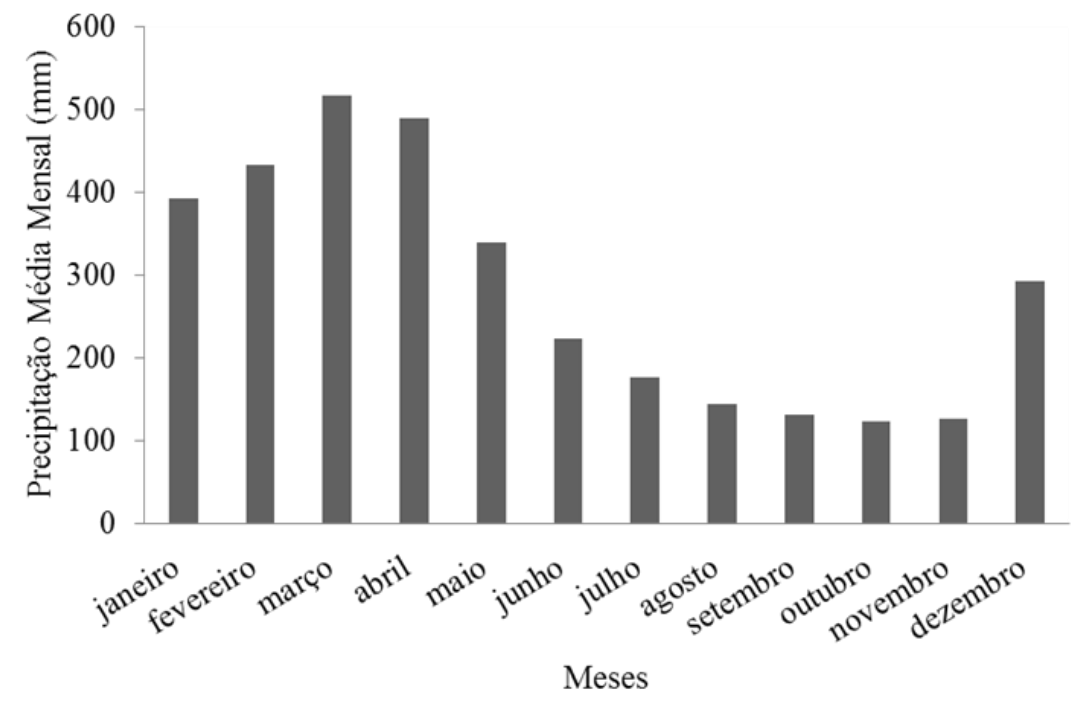

Figura 8 - Média mensal em mm dia-1 de 2003 a 2015 para Belém-PA.

\section{CONCLUSÃO}

A partir dos resultados obtidos neste estudo, pode-se concluir que:

A "famosa chuva da tarde" em Belém, tem grande contribuição para os totais mensais, sendo as $1600 \mathrm{HL}$ o horário de maior contribuição. No período entre a madrugada e inicio da manhã, os valores de contribuição são mínimos.

Quanto à contribuição nos meses mais chuvosos (dezembro a maio), o mês de dezembro, apresentou contribuição de $26 \%$ em relação ao total mensal no horário das $1600 \mathrm{HL}$ e a menor foi no mês de maio, com 14\%. Foi possível perceber que nos meses de dezembro a março esta contribuição fica restrita ao período da tarde. Já nos meses de abril e maio, nota-se que as mesmas aumentam um pouco no período da noite. Isso ainda é visto nos meses menos chuvosos (junho a agosto) e volta a ficar no período da tarde de setembro a novembro. Esta mudança esta relacionada com a atuação da ZCIT e pelos efeitos de meso escala como LI associada as BM e também a formação de BF.

O aumento das contribuições se deve ao deslocamento da ZCIT para norte, e também a formação de SCM, no período da noite, além de MLF com maior frequência no mês de agosto, assim como efeitos locais, como BM e DOL.

Nos anos de 2006 a 2013 estiveram entre os anos de maiores e menores volumes de precipitações anuais, respectivamente, porém, nesses anos o fenômeno ENOS estava em neutralidade, indicando a influencia de outro padrão, como a TSM do oceano Atlântico.

\section{AGRADECIMENTOS}

Este trabalho é parte integrante da dissertação de mestrado do Programa de Pós-Graduação em Meteorologia (PPGMET) da Universidade Federal de 
Campina Grande (UFCG) e contou com a colaboração do $2^{\circ}$ Distrito de Meteorologia (2 DISME) do Instituto Nacional de Meteorologia (INMET), instituição de reconhecida relevância no cenário regional e nacional, na qual os autores agradecem pelos dados repassados, item essencial para a elaboração desta pesquisa.

\section{REFERÊNCIAS BIBLIOGRÁFICAS}

ALCÂNTARA, C.R.; SILVA DIAS, M.A.F.; SOUZA, E.P.; COHEN, J. C. P. Verification of the Role of the Low Level Jets in Amazon Squall Lines. Atmospheric Research, v. 100, p. 36-44, 2011.

ALCÂNTARA, C.R.; SOUZA, E.P.; SILVA DIAS, M.A.F.; BIAZETO, B. Influência dos jatos de médios e baixos níveis nos processos de nuvem: Estudo numérico de uma linha de instabilidade Amazônica. Revista Brasileira de Meteorologia, v. 29, n. 1, 29-46, 2014.

ALBUQUERQUE, M.F.; SOUZA, E.B.; OLIVEIRA, M.C.F.; SOUZA JR, J.A. Precipitação nas mesorregiões do Estado do Pará: Climatologia, variabilidade e tendência nas últimas décadas (1978-2008). Revista Brasileira de Climatologia. ISSN: 1980-55X, v.6, 2010.

ANDREOLI, R.V.; KAYANO, M.T. Multi-scale variability of sea surface temperature in the tropical Atlantic. J. Geophys. Res., v.109, C05009, doi: 10.1029/2003JC002220, 2004.

ANDREOLI, R.V.; KAYANO, M.T. A importância relativa do Atlântico Tropical Sul e Pacífico Leste na variabilidade de precipitação do Nordeste do Brasil. Revista Brasileira de Meteorologia, v. 22, n.1, p.63-74, 2007.

ASNANI, G.C. Tropical Meteorology. Pune, Índia: Noble Printers, 2v, 121p. 1993.

BASTOS, T.X.; PACHECO, N.A.; NECHET, D.; ABREU, T.D. Aspectos Climáticos de Belém nos Últimos 100 anos, Boletim da Pesquisa e Desenvolvimento. Documentos. EMBRAPA-CPATU, Belém-Pará, 2002.

CAVALCANTE, I.F.A.; FERREIRA, N.J.; SILVA DIAS, M.A.F.; JUSTI, M.G.A. Tempo e clima no Brasil. São Paulo: Oficina de Textos, 2009.

COHEN, J.C.P. Um estudo observacional de linhas de instabilidade na Amazônia. Dissertação de Mestrado. Programa de Pós-Graduação em MeteorologiaInstituto Nacional de Pesquisas. São José dos Campos, 153p. 1989.

COHEN, J.C.P.; SILVA DIAS, M. A. F.; NOBRE, C. Environmental conditions associated with Amazonian squall lines: A case study. Monthly Weather Review, 123, 3163-3174, 1995.

CPC/NOAA. Cold \& Warm Episodes by Season. Climate Prediction Center Internet Team. Disponível em: 04 maio 2010. Acesso em 06 jun 2016.

DE SOUZA, E.B.; AMBRIZZI, T. Modulation of the intraseasonal rainfall over tropical Brazil by the Madden-Julian oscillation. International Journal of Climatology. São Paulo, v.26, p.1759-1776, 2006.

FERREIRA, D.B.S.; DE SOUZA, E.B.; DE MORAES, B.C.; FILHO, L.G.M. Spatial and Temporal Variability of Rainfall in Eastern Amazon during the Rainy Season. The Scientific Word Journal, Volume 2015. Article ID 209783, 9 pages, 2015. 
FIGUEIREDO, C.T.; MOTA, M.A.S. Análise da precipitação na região metropolitana de Belém (PA) ao longo de um ano (agosto/2008 a julho/2009). XVI Congresso de Meteorologia. Anais. 2010.

FIGUEROA, A.S.N.; NOBRE, C. Precipitation distribution over central and estern tropical South America. Climanálise, v. 5, n. 6, p.36-45. 1990.

INMET, Normais Climatológicas (1961-1990), INMET, Brasília-DF, 1992.

JANOWIAK, J.E, KOUSKY, V.E, JOYCE, R.J. Diurnal cycle of precipitation determined from the CMORPH high spatial and temporal resolution global precipitation analyses. Journal of Geophysical Research. Atmospheres 110(D23): D23105, doi: 10.1029/2005JD006156. 2005.

KAYANO, M.T.; CAPISTRANO, V.B. How the Atlantic multidecadal oscillation (AMO) modifies the ENSO influence on the South American rainfall. International Journal of Climatology, v. n/a, p. n/a-n/a, 2013.

KOUSKY, V.E. Diurnal rainfall variation in the northeast Brazil. Mon. Wea. Rev. 108, 488-498, 1980.

KOUSKY, V.E.; KAGANO, M.T. A climatological study of the tropospheric circulation over the Amazon region. Acta Amazon, v.11, n.4, p.743-753. 1981.

LIEBMANN, B.; HEBDON, H.H. Synoptic-scale disturbance near the equator. Journal Atmospheric Sciences, 47, 1463-1479p. 1990.

LOUREIRO, R.S.; SARAIVA, J.M.; SARAIVA, I ; SENNA, R.C. ; FREDÓ, A.S. Estudo dos eventos extremos de precipitação ocorridos em 2009 no estado do Pará. Revista Brasileira de Meteorologia (Impresso), v. 29, p. 83-94, 2014.

MACHADO, L.A.T.; SILVA DIAS, M.A.F.; MORALES, C.; FISCH, G.; VILA, D.; ALBRECHT, R.; GOODMAN, S. J.; CALHEIROS, A.J.P.; BISCARO, T.; KUMMEROW, C.; COHEN, J.C.P.; FITZJARRALD, D.; NASCIMENTO, E. L.; SAKAMOTO, M. S.; CUNNINGHAM, C.; CHABOUREAU, J.P.; PETERSEN, W. A.; ADAMS, D. K.; BALDINI, L.; ANGELIS, C.F.; SAPUCCI, L.F.; SALIO, P.; BARBOSA, H.M.J.; LANDULFO, E.; SOUZA, R.A.F.; BLAKESLEE, R.J.; BAILEY, J.; FREITAS, S.; LIMA, W.F.A.; TOKAY, A. The Chuva Project How Does Convection Vary Across Brazil? American Meteorological. Society.Sept. 2014.

MATOS, A.P.; COHEN, J.C.P. Circulação de brisa e a banda de precipitação na margem leste da baía de Marajó. Ciência e Natura, V. 38, Ed. Especial-IX Workshop Brasileiro de Micro meteorologia, p. 21-27, 2016.

MOTA, G.V. Estudo observacional de distúrbios ondulatórios de leste no Nordeste Brasileiro. São Paulo. Dissertação de Mestrado, 1997.

MONTEIRO, L. D. A.; MOTA, M. A. S. Análise da variação da temperatura e precipitação em Belém em anos de El Niño e La Niña. In: XVI Congresso Brasileiro de Meteorologia, 2010, Belém-PA. A Amazônia e o Clima Global, 2010.

NAGHETTINI, M.; PINTO, E.J.A. Hidrologia Estatística. Belo Horizonte: CPRM/SGB, 2007.

NECHET, D. Variabilidade diurna da precipitação em Belém-Pa. Anais do $3^{\circ}$ Congresso Brasileiro de Meteorologia, p.204-211. 1984 a. 
NECHET, D. Variabilidade diurna da precipitação em Belém-PA, In: CONGRESSO BRASILEIRO DE METEOROLOGIA, 3., 1984, Belo Horizonte. Anais. Rio de Janeiro: SBM, p.204-211. 1984b.

NECHET. D. Variabilidade diurna de precipitação em Belém-PA: aplicação em planejamento a médio e longo prazo. Boletim Climatológico. Presidente Prudente, SP, v.2, n.3, p.223-227. 1997.

NIMER, E. Climatologia do Brasil. $2^{\circ}$ edição. Rio de Janeiro. Fundação Instituto Brasileiro de Geografia e Estatística. IBGE, 1989.

OLIVEIRA, L.L. et al. Estudo do Comportamento da Precipitação Pluviométrica nos diversos períodos do dia em Belém-PA. In: I Simpósio de Recursos Hídricos da Amazônia, Manaus-AM, Núcleo de Hidro meteorologia, SECTAM, 2003.

OLIVEIRA, G.B; ALCANTARA, C.R, SOUZA, E.P. Caracterização de perfil de vento no ambiente de formação das linhas de instabilidade amazônicas. Ciência e Natura, Santa Maria v.38 n.1, Jan.- Abr. p. 393 - 403, DOI:10.5902/2179460X18337. 2016.

SANTOS, O.C.O. Análise do uso do solo e dos recursos hídricos da micro bacia hidrográfica do Igarapé Apeú, nordeste do Estado do Pará. Tese de Doutorado. Programa de Pós-Graduação em Geografia. Universidade Federal do Rio de Janeiro. Rio de Janeiro, 269p. 2006.

SODRÉ, G.R. Estudo da convecção de Mesoescala em Diferentes Superfícies na Amazônia Oriental. Dissertação (Mestrado) - Universidade Federal do Pará, Belém, 2013.

SOUZA, E.B.; KAYANO, M.T.; TOTA, J.; PEZZI, L.; FISCH, G.; NOBRE, C. One the influence of El Niño, La Niña and Atlantic Dipole pattern the Amazonian rainfall during 1960 - 1998, Acta Amazonica, v. 30, p. 305 - 318, 2000. 